Elsevier required licence: (C) <2017>. This manuscript version is made available under the CC-BY-NC-ND 4.0 license http://creativecommons.org/licenses/by-nc-nd/4.0/ 


\section{Fate of trace organic contaminants in oxic-settling-anoxic (OSA) process applied for biosolids reduction during wastewater treatment}

\section{Supplementary data}

Galilee U. Semblante a , Faisal I. Hai ${ }^{\text {a* }}$, James McDonald ${ }^{\text {b }}$, Stuart J. Khan ${ }^{\text {b }}$, Mark Nelson ${ }^{\text {c }}$, Duu-Jong Lee ${ }^{\mathrm{d}}$, William E. Price ${ }^{\mathrm{e}}$, and Long D. Nghiem ${ }^{\mathrm{a}}$

${ }^{a}$ Strategic Water Infrastructure Laboratory, School of Civil, Mining and Environmental Engineering, University of Wollongong, Wollongong, NSW 2522, Australia

${ }^{\mathrm{b}}$ Water Research Centre, The University of New South Wales, Sydney NSW 2052, Australia

${ }^{c}$ School of Mathematics and Applied Statistics, University of Wollongong, Wollongong, NSW 2522, Australia

${ }^{\mathrm{d}}$ Department of Chemical Engineering, National Taiwan University, Taipei, Taiwan

e Strategic Water Infrastructure Laboratory, School of Chemistry, University of Wollongong, Wollongong, NSW 2522, Australia

* Corresponding author:

Email: faisal@uow.edu.au, Ph: +61 242213054

Table S1. The basic properties of domestic sewage where $n=$ number of samples

\begin{tabular}{ccc}
\hline Property & Average & $n$ \\
\hline tCOD & $474 \pm 292 \mathrm{mg} / \mathrm{L}$ & 72 \\
\hline sCOD & $101 \pm 54 \mathrm{mg} / \mathrm{L}$ & 72 \\
\hline $\mathrm{TOC}$ & $47.2 \pm 23.5 \mathrm{mg} / \mathrm{L}$ & 70 \\
\hline $\mathrm{TN}$ & $45.0 \pm 11.1 \mathrm{mg} / \mathrm{L}$ & 70 \\
\hline $\mathrm{NH}_{4}{ }^{-}-\mathrm{N}$ & $78.4 \pm 32.1 \mathrm{mg} / \mathrm{L}$ & 69 \\
\hline $\mathrm{PO}_{4}{ }^{--}-\mathrm{P}$ & $30.3 \pm 14.7 \mathrm{mg} / \mathrm{L}$ & 69 \\
\hline $\mathrm{pH}$ & $7.2 \pm 0.5$ & 61 \\
\hline
\end{tabular}


Table S2. Sample preparation, solid phase extraction, and TrOC analysis

Table S2-a. Method description

Sample preparation. All samples (influent, effluent, and sludge) were initially centrifuged at $3720 \mathrm{xg}$ and $4{ }^{\circ} \mathrm{C}$ for $10 \mathrm{~min}$ (Beckman Coulter, USA). To obtain TrOC concentration in the aqueous phase, the supernatant (from wastewater and sludge samples) was diluted to $500 \mathrm{~mL}$ in MilliQ water, and then sequentially filtered using $1 \mu \mathrm{m}$ and $0.7 \mu \mathrm{m}$ glass fibre filter papers. The filtered liquid later underwent solid phase extraction (SPE). To obtain TrOC concentration in the solid phase of sludge, the pellet (from sludge samples only) was freeze-dried (Christ $\mathrm{GmbH}$, Germany) for $12 \mathrm{~h}$. The dried sample was ground to powder using mortar and pestle, and then $0.5 \mathrm{~g}$ of powder was placed in a capped glass vial. In the first round of extraction, the powder was re-suspended in $10 \mathrm{~mL}$ of methanol, vortexed (Ratek, Australia), and then ultrasonicated for $10 \mathrm{~min}$ at $40{ }^{\circ} \mathrm{C}$. The mixture was centrifuged at $3720 \mathrm{xg}$ for $10 \mathrm{~min}$, and then the supernatant was decanted and set aside. In the second round of extraction, the pellet from the previous extraction was re-suspended in $10 \mathrm{~mL}$ of dichloromethane and ethanol mixture $(1: 1 \mathrm{v} / \mathrm{v})$, vortexed (Ratek, Australia), and then ultrasonicated for $10 \mathrm{~min}$ at $40{ }^{\circ} \mathrm{C}$. The mixture was centrifuged at $3720 \mathrm{xg}$ for $10 \mathrm{~min}$, and then the supernatant was decanted and added to the previous extract. The combined extract was diluted to $500 \mathrm{~mL}$ with Milli-Q water, and then sequentially filtered using $1 \mu \mathrm{m}$ and $0.7 \mu \mathrm{m}$ glass fibre filter papers. These samples later underwent solid phase extraction (SPE).

Surrogate TrOC standards. All analytes had isotopically labelled standards except for metoprolol, benzotriazole, benzophenone, saccharin, oxybenzone, fenbofibrate, allopurinol, acesulfame, cyclamate, sucralose, aspartame, clofibric acid, dichloroprop, propylparaben, phenylphenol, and butylparaben. Those with standards were quantified using the isotope dilution method. Those without standards were determined using external calibration.

Solid phase extraction. Prior to SPE, the samples were spiked with $50 \mu \mathrm{L}$ of a $1 \mathrm{mg} / \mathrm{L}$ solution of isotopically labelled surrogate TrOC standards (Supplementary Table S3) and mixed thoroughly. Then, the samples were loaded onto hydrophilic/lipophilic Oasis HLB cartridges (Waters, USA) that have had been sequentially conditioned with $5 \mathrm{~mL}$ of methyltert-butyl ether, $5 \mathrm{~mL}$ of methanol, and twice with $5 \mathrm{~mL}$ of Milli-Q water. The loading rate (15 $\mathrm{mL} / \mathrm{min}$ ) was controlled by adjusting the vacuum pressure in the SPE manifold. After loading, the cartridges were rinsed with $5 \mathrm{~mL}$ of MilliQ, gently dried using $\mathrm{N}_{2}$ gas, and then stored in a sealed bag at $4^{\circ} \mathrm{C}$ until elution and analysis.

Liquid Chromatography. Analytes were separated using an Agilent (Palo Alto, CA, USA) 1200 series high performance liquid chromatography (HPLC) system equipped with a $150 \mathrm{x}$ $4.6 \mathrm{~mm}, 5 \mu \mathrm{m}$ particle size, Luna C18 (2) column (Phenomenex, Torrence CA, USA) . A binary gradient consisting of $5 \mathrm{mM}$ ammonium acetate in water (A) and $100 \%$ methanol (B) at a flow rate of $800 \mu \mathrm{L} \mathrm{min}{ }^{-1}$ was used. For electrospray ionization (ESI) positive analyses, the gradient was as follows: $10 \% \mathrm{~B}$ held for $0.50 \mathrm{~min}$, stepped to $50 \% \mathrm{~B}$ at $0.51 \mathrm{~min}$ and increased linearly to $100 \% \mathrm{~B}$ at $8 \mathrm{~min}$, then held at $100 \% \mathrm{~B}$ for $2 \mathrm{~min}$. For ESI negative analyses, the 
gradient was as follows: $10 \% \mathrm{~B}$ held for $0.50 \mathrm{~min}$, stepped to $60 \% \mathrm{~B}$ at $0.51 \mathrm{~min}$ and increased linearly to $100 \%$ B at $8 \mathrm{~min}$, then held at $100 \%$ B for $3 \mathrm{~min}$. A 5 min equilibration step at $10 \%$ $\mathrm{B}$ was used at the beginning of each run. For atmospheric pressure chemical ionization (APCI) analysis the eluants consisted of milli-Q grade water (A) and $0.1 \% \mathrm{v} / \mathrm{v}$ formic acid in methanol with the following ramp at a flow rate of $700 \mu \mathrm{L} \mathrm{min}^{-1} .60 \% \mathrm{~B}$ held for $5 \mathrm{~min}$, increased linearly to $100 \%$ B at $20 \mathrm{~min}$, then held at $100 \%$ B for $3 \mathrm{~min}$. A 3 min equilibrium step preceded injection. An injection volume of $10 \mu \mathrm{L}$ was used for all methods. Analytical methods using ESI and APCI are based on that of Vanderford et al. (2006) and Vanderford et al. (2003), respectively.

Mass Spectrometry. Mass spectrometry was performed using an API 4000 triple quadrupole mass spectrometer (Applied Biosystems, Foster City, CA, USA) equipped with a turbo-V ion source employed in both positive and negative electro-spray modes. Steroids were analysed the source configured for (APCI) in positive mode. Using multiple reaction monitoring (MRM) two mass transitions for all but three of the analytes were monitored for unequivocal confirmation. One mass transition for the labeled internal standard was monitored. Only the first transition was used for quantitiation. Relative retention times of the analyte and isotopically labeled internal standard were also monitored to ensure correct identification.

Calibration and limits of Detection. Standard solutions of all analytes were prepared at 1, 5, $10,50,100,500$ and $1000 \mathrm{ng} / \mathrm{mL}$. A relative response ratio of analyte/internal standard over a $1-1000$ ng concentration range was generated enabling quantitation with correction for losses due to ion suppression and incomplete SPE recovery. All calibration curves had a correlation coefficient of 0.99 or better. Detection limits were defined as the concentration of an analyte giving a signal to noise $(\mathrm{s} / \mathrm{n})$ ratio greater than 3 . The Limits of Reporting were determined using a $\mathrm{s} / \mathrm{n}$ ratio of greater than 10 .

\section{References:}

[1] Vanderford, B.J., Snyder, S.A. 2006. Analysis of Pharmaceuticals in Water by Isotope Dilution Liquid Chromatography/Tandem Mass Spectrometry. Environ Sci Technol, 40(23), 7312-7320.

[2] Vanderford, B.J., Pearson, R.A., Rexing, D.J., Snyder, S.A. 2003. Analysis of Endocrine Disruptors, Pharmaceuticals, and Personal Care Products in Water Using Liquid Chromatography/Tandem Mass Spectrometry. Anal Chem, 75(22), 6265-6274.

Table S2-b. Transitions for compounds using ESI positive mode

$\begin{array}{lll}\text { Compound } & \begin{array}{l}\text { Precursor Ion } \\ \left(\mathrm{m} \mathrm{z}^{-1}\right)\end{array} & \begin{array}{l}\text { Product Ion } \\ \left(\mathrm{m} \mathrm{z}^{-1}\right)\end{array} \\ \text { paracetamol } & 152.1 & 110.1 \\ \text { paracetamol-15N13C } & 155.0 & 111.0 \\ \text { sulfamethoxazole-1 } & 254.0 & 156.1 \\ \text { sulfamethoxazole-2 } & 254.0 & 92.0 \\ \text { sulfamethoxazole-D4 } & 258.1 & 160.1\end{array}$




$\begin{array}{lll}\text { caffeine-1 } & 195.0 & 138.1 \\ \text { caffeine-2 } & 195.0 & 110.1 \\ \text { caffeine-D9 } & 204.1 & 144.2 \\ \text { trimethoprim-1 } & 291.1 & 230.2 \\ \text { trimethoprim-2 } & 291.1 & 261.1 \\ \text { trimethoprim-D9 } & 300.3 & 234.2 \\ \text { benzotriazole } & 120.0 & 63.5 \\ \text { benzotriazole D4-1 } & 124.0 & 67.9 \\ \text { aspartame-294-1 } & 295.1 & 119.8 \\ \text { aspartame-294-2 } & 295.1 & 179.8 \\ \text { triamterene-1 } & 254.2 & 237.0 \\ \text { triamterene-2 } & 254.2 & 104.0 \\ \text { triamterene-D5-1 } & 259.2 & 242.2 \\ \text { primidone-1 } & 219.2 & 162.2 \\ \text { primidone-2 } & 219.2 & 119.0 \\ \text { primidone-D5-1 } & 224.2 & 167.0 \\ \text { carazolol-1 } & 299.1 & 115.6 \\ \text { carazolol-2 } & 299.1 & 221.6 \\ \text { carazolol-D7-1 } & 306.2 & 122.8 \\ \text { carazolol-D7-2 } & 306.2 & 221.6 \\ \text { meprobamate-1 } & 218.9 & 158.2 \\ \text { meprobamate-2 } & 218.9 & 115.1 \\ \text { meprobamate-D3-1 } & 221.9 & 161.2 \\ \text { bisoprolol-1 } & 326.2 & 115.8 \\ \text { bisoprolol-2 } & 326.2 & 73.5 \\ \text { bisoprolol-D-5-1 } & 331.2 & 120.8 \\ \text { enalapril-1 } & 377.1 & 234.1 \\ \text { enalapril-2 } & 377.1 & 91.1 \\ \text { enalapril-D5 } & 382.2 & 239.2 \\ \text { TCEP-1 } & 284.9 & 62.9 \\ \text { TCEP-2 } & 284.9 & 223.0 \\ \text { TCEP-D12-1 } & 297.0 & 66.7 \\ \text { TCEP-D12-2 } & 297.0 & 231.6 \\ \text { dilantin-1 } & 253.1 & 182.1 \\ \text { dilantin-2 } & 253.1 & 104.1 \\ \text { dilantin-D10 } & 263.1 & 192.2 \\ \text { simazine-1 } & 202.1 & 132.1 \\ \text { simazine-2 } & 202.1 & 124.1 \\ \text { simazineD10-1 } & 212.2 & 137.1 \\ \text { carbamazepine-1 } & 237.0 & 194.2 \\ \text { carbamazpine-2 } & 237.0 & 192.1 \\ \text { carbamazpine-D10 } & 247.1 & 204.3 \\ \text { omeprazole-1 } & 346.2 & 198.2 \\ \text { omeprazole-2 } & 346.2 & 136.1 \\ \text { omeprazole-D3 } & 349.2 & 19.0 \\ \text { DEET-1 } & & \end{array}$




$\begin{array}{lll}\text { DEET-2 } & 192.2 & 108.9 \\ \text { DEET-D7-1 } & 199.2 & 126.1 \\ \text { atrazine-1 } & 216.0 & 174.2 \\ \text { atrazine-2 } & 216.0 & 96.1 \\ \text { atrazine-D5 } & 221.3 & 179.1 \\ \text { verapamil-1 } & 455.4 & 165.1 \\ \text { verapamil-2 } & 455.4 & 150.0 \\ \text { verapamil-D6-1 } & 461.4 & 165.2 \\ \text { linuron-1 } & 249.0 & 182.2 \\ \text { linuron-2 } & 249.0 & 160.1 \\ \text { linuron-D6 } & 255.0 & 160.1 \\ \text { diazepam-1 } & 285.1 & 193.1 \\ \text { diazepam-2 } & 285.1 & 154.2 \\ \text { diazepam-D5-1 } & 290.1 & 198.1 \\ \text { benzophenone-1 } & 183.0 & 104.2 \\ \text { benzophenone-2 } & 183.0 & 76.5 \\ \text { benzophenone D10 } & 193.1 & 109.0 \\ \text { saccharin } & 183.0 & 76.7 \\ \text { saccharin d4 } & 183.1 & 104.0 \\ \text { clozapine-1 } & 327.1 & 270.2 \\ \text { clozapine-2 } & 327.1 & 192.1 \\ \text { clozapine-D4-1 } & 331.2 & 272.0 \\ \text { amtriptyline-1 } & 278.2 & 233.0 \\ \text { amtriptyline-2 } & 278.2 & 117.1 \\ \text { amtriptyline-D6-1 } & 284.4 & 233.1 \\ \text { TCPP d18 } & 344.1 & 326.4 \\ \text { hydroxyzine-1 } & 375.3 & 201.1 \\ \text { hydroxyzine-2 } & 375.3 & 165.1 \\ \text { hydroxyzine-D8-1 } & 383.3 & 201.1 \\ \text { oxybenzone-1 } & 229.1 & 151.0 \\ \text { oxybenzone-2 } & 229.1 & 104.7 \\ \text { Diazinon-1 } & 305.1 & 169.1 \\ \text { Diazinon-2 } & 305.1 & 115.0 \\ \text { DiazinonD10-1 } & 315.2 & 170.1 \\ \text { fenofibrate-1 } & 361.0 & 232.8 \\ \text { fenofibrate-2 } & 361.0 & 138.8 \\ \text { Chlorpyrifos-1 } & 351.9 & 96.2 \\ \text { Chlorpyrifos-2 } & 349.9 & 197.9\end{array}$

Table S2-c. Transitions for compounds using ESI negative mode

$\begin{array}{lll}\text { Compound } & \begin{array}{l}\text { Precursor Ion } \\ \left(\mathrm{m} \mathrm{z}^{-1}\right)\end{array} & \begin{array}{l}\text { Product Ion } \\ \left(\mathrm{m} \mathrm{z}^{-1}\right)\end{array} \\ \text { saccharin 1 } & 182.1 & 41.8 \\ \text { saccharin 2 } & 182.1 & 105.3 \\ \text { saccharin D4 1 } & 185.9 & 42.0\end{array}$




\begin{tabular}{|c|c|c|}
\hline saccharin D4 2 & 185.9 & 105.9 \\
\hline acesulfame-1 & 161.8 & 81.8 \\
\hline acesulfame-2 & 161.8 & 77.9 \\
\hline salicyclic acid -1 & 136.8 & 93.0 \\
\hline salicyclic acid -2 & 136.8 & 64.7 \\
\hline salicilicAcidD6 & 140.9 & 96.9 \\
\hline cyclamate-1 & 177.9 & 79.4 \\
\hline cyclamate-2 & 177.9 & 80.3 \\
\hline cyclamate- D11 & 188.9 & 79.2 \\
\hline sucralose-1 & 398.9 & 36.7 \\
\hline sucralose-2 & 398.9 & 360.4 \\
\hline aspartame-2 & 293.0 & 199.0 \\
\hline aspartame-1 & 293.0 & 260.8 \\
\hline ketoprofen-1 & 252.8 & 208.8 \\
\hline ketoprofen-2 & 252.8 & 196.7 \\
\hline ketoprofen-D3 & 255.6 & 211.7 \\
\hline naproxen-1 & 228.9 & 184.6 \\
\hline naproxen-2 & 228.9 & 169.8 \\
\hline naproxen-D3 & 231.9 & 187.8 \\
\hline bisphenol A-1 & 226.9 & 211.8 \\
\hline bisphenol A-2 & 226.9 & 132.9 \\
\hline bisphenol A-D6 & 232.9 & 214.9 \\
\hline diclofenac-1 & 293.9 & 249.7 \\
\hline diclofenac-2 & 293.9 & 213.7 \\
\hline diclofenac-D4-1 & 297.9 & 253.8 \\
\hline propylparaben-1 & 178.9 & 92.0 \\
\hline propylparaben-2 & 178.9 & 136.2 \\
\hline diuron-1 & 230.8 & 185.4 \\
\hline diuron-2 & 230.8 & 149.7 \\
\hline diuronD6-1 & 236.9 & 185.8 \\
\hline Ibuprofen-1 & 204.9 & 160.8 \\
\hline Ibuprofen-2 & 204.9 & 158.8 \\
\hline ibuprofen-D3 & 208.0 & 163.9 \\
\hline phenylphenol-1 & 168.9 & 114.8 \\
\hline phenylphenol-2 & 168.9 & 140.8 \\
\hline gemfibrozil-1 & 248.9 & 120.5 \\
\hline gemfibrozil-2 & 248.9 & 126.8 \\
\hline gemfibrozil-D6 & 254.9 & 120.9 \\
\hline triclocarban-1 & 312.8 & 159.8 \\
\hline triclocarban-2 & 312.8 & 125.8 \\
\hline triclocarban-D4-1 & 317.0 & 159.8 \\
\hline Triclosan-1 & 286.6 & 35.0 \\
\hline Triclosan-2 & 286.7 & 141.6 \\
\hline Triclosan-D3 & 289.7 & 34.9 \\
\hline t-octylphenol-1 & 204.9 & 133.0 \\
\hline t-octylphenol-2 & 205.2 & 132.9 \\
\hline
\end{tabular}




$\begin{array}{lll}\text { n-octylphenol-D17 } & 222.1 & 108.0 \\ \text { nonylphenol-1 } & 219.0 & 106.0 \\ \text { nonylphenol-D4-1 } & 223.1 & 110.0 \\ \text { PFOA 1 } & 413.0 & 368.5 \\ \text { PFOA 2 } & 413.0 & 168.7 \\ \text { PFOA 13C8 } & 421.0 & 375.8 \\ \text { PFOS 1 } & 499.0 & 79.8 \\ \text { PFOS 2 } & 499.0 & 98.7 \\ \text { PFOS 13C8 } & 506.9 & 79.9 \\ \text { butylparaben-1 } & 192.9 & 91.8 \\ \text { butylparaben 2 } & 192.9 & 135.9\end{array}$

Table S2-d. Transitions for compounds using APCI positive mode

Compound

Estriol 1

Estriol 2

Estriol-D2

Androstendione 1

Androstendione 2

Androstendione-D3

Etiocholanolone 1

Etiocholanolone 2

Etiocholanolone-D2

Androsterone 1

Androsterone 2

Estrone 1

Estrone 2

Estrone-D4

$17 \beta$-Estradiol 1

$17 \beta$-Estradiol 2

17 $\beta$-Estradiol-D4

$17 \alpha$-Estradiol 1

$17 \alpha$-Estradiol 2

$17 \alpha$-Ethynylestradiol 1

$17 \alpha$-Ethynylestradiol 2

$17 \alpha$-Ethynylestradiol-D4

Testosterone 1

Testosterone 2

Testosterone-D2

$\begin{array}{ll}\begin{array}{l}\text { Precursor Ion } \\ \left(\mathrm{m} \mathrm{z}^{-1}\right)\end{array} & \begin{array}{l}\text { Product Ion } \\ \left(\mathrm{m} \mathrm{z}^{-1}\right)\end{array} \\ 271.1 & 253.1 \\ 271.1 & 133.0 \\ 273.2 & 255.2 \\ 287.2 & 97.1 \\ 287.2 & 109.2 \\ 290.2 & 100.1 \\ 273.2 & 255.3 \\ 273.2 & 91.1 \\ 275.2 & 257.1 \\ 273.2 & 255.2 \\ 273.2 & 91.0 \\ 271.2 & 159.2 \\ 271.2 & 133.0 \\ 275.1 & 161.0 \\ 255.2 & 159.3 \\ 255.2 & 133.2 \\ 259.1 & 161.1 \\ 255.2 & 159.3 \\ 255.2 & 133.2 \\ 279.2 & 133.1 \\ 279.2 & 159.2 \\ 283.1 & 135.1 \\ 289.2 & 97.2 \\ 289.2 & 109.1 \\ 291.2 & 99.1\end{array}$


Table S3. Summary of TrOCs that were analysed and detected in the influent at different sampling periods. All analytes have isotopically-labelled surrogate standards except for those listed in Table S2. TrOC sampling and analysis were performed when SRTsBRs was maintained at $10 \mathrm{~d}$ and SRText was varied (10-40 d). The sampling campaigns occurred at different seasons.

\begin{tabular}{|c|c|c|c|c|c|c|c|c|c|c|}
\hline \multirow[b]{3}{*}{ TrOC } & \multirow[b]{3}{*}{ Chemical Structure } & \multirow{3}{*}{$\begin{array}{c}\log \\
\mathrm{D} \\
(\mathrm{pH} \\
7 ; \\
\left.25^{\circ} \mathrm{C}\right) \\
\end{array}$} & \multirow{3}{*}{$\begin{array}{c}\text { Type or } \\
\text { application }\end{array}$} & \multirow{3}{*}{$\begin{array}{c}\text { Detection } \\
\text { limit } \\
(\mathrm{ng} / \mathrm{L})\end{array}$} & \multicolumn{6}{|c|}{$\operatorname{SRT}_{\text {ext }}(\mathrm{d}) /$ season } \\
\hline & & & & & \multicolumn{2}{|c|}{40 / winter } & \multicolumn{2}{|c|}{$20 /$ spring } & \multicolumn{2}{|c|}{10 / summer } \\
\hline & & & & & Analysed & $\begin{array}{l}\text { Detected } \\
\text { in } \\
\text { influent }\end{array}$ & Analysed & $\begin{array}{l}\text { Detected } \\
\text { in } \\
\text { influent }\end{array}$ & Analysed & $\begin{array}{c}\text { Detecte } \\
\mathrm{d} \text { in } \\
\text { influent }\end{array}$ \\
\hline Acesulfame & & -2.88 & $\begin{array}{l}\text { Artificial } \\
\text { sweetener }\end{array}$ & 5 & No & - & Yes & No & Yes & No \\
\hline Cyclamate & & -2.46 & $\begin{array}{l}\text { Artificial } \\
\text { sweetener }\end{array}$ & 5 & No & - & Yes & No & Yes & No \\
\hline Atenolol & & -2.09 & $\begin{array}{c}\text { Pharmaceutical } \\
\text { (beta-blocker) }\end{array}$ & 5 & Yes & Yes & Yes & Yes & Yes & Yes \\
\hline Aspartame & & -1.99 & $\begin{array}{l}\text { Artificial } \\
\text { sweetener }\end{array}$ & 5 & No & - & Yes & Yes & Yes & Yes \\
\hline Salicylic acid & $\mathrm{OH}$ & -1.13 & Pharmaceutical & 5 & No & - & Yes & No & Yes & Yes \\
\hline
\end{tabular}




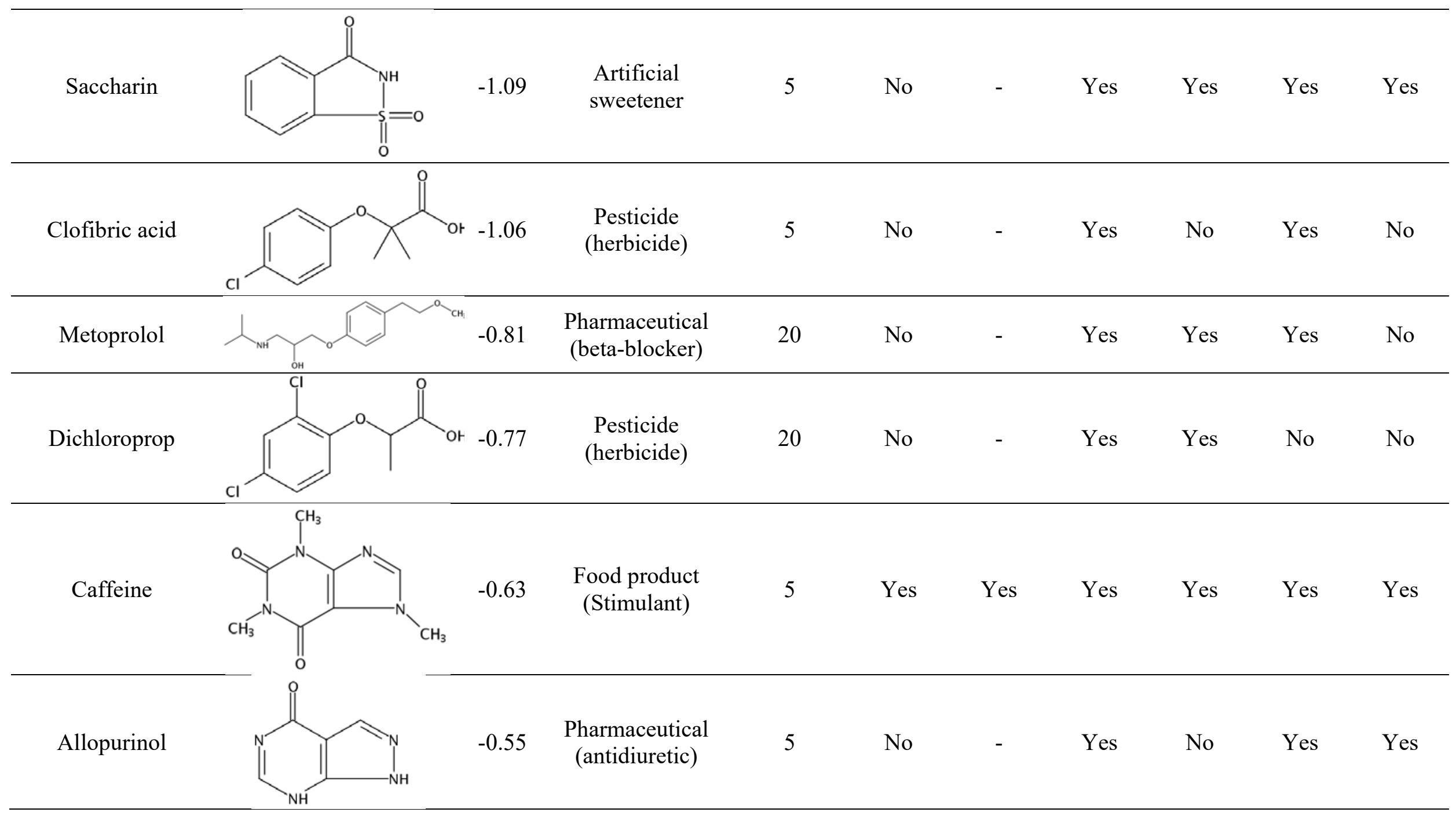




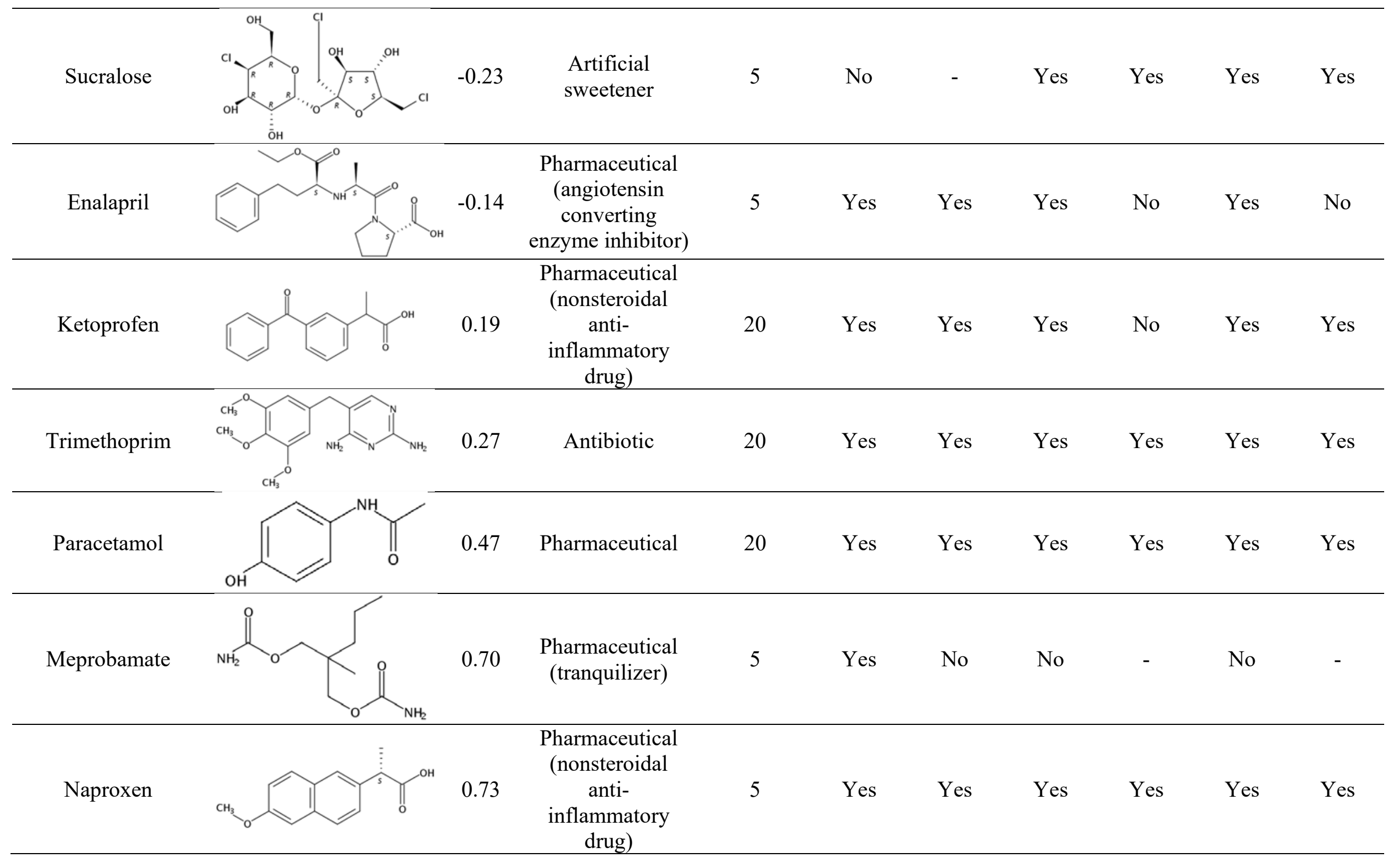




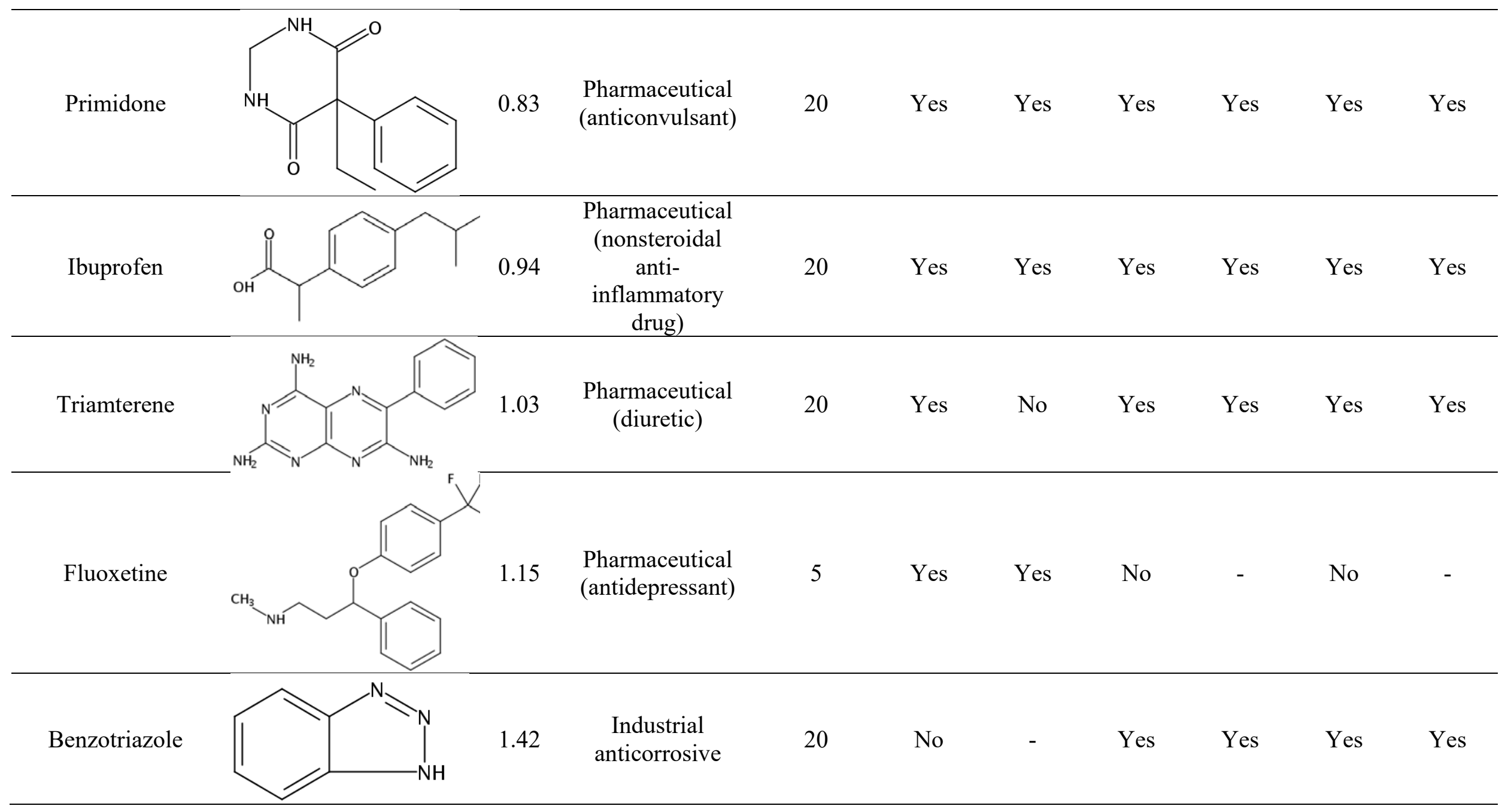




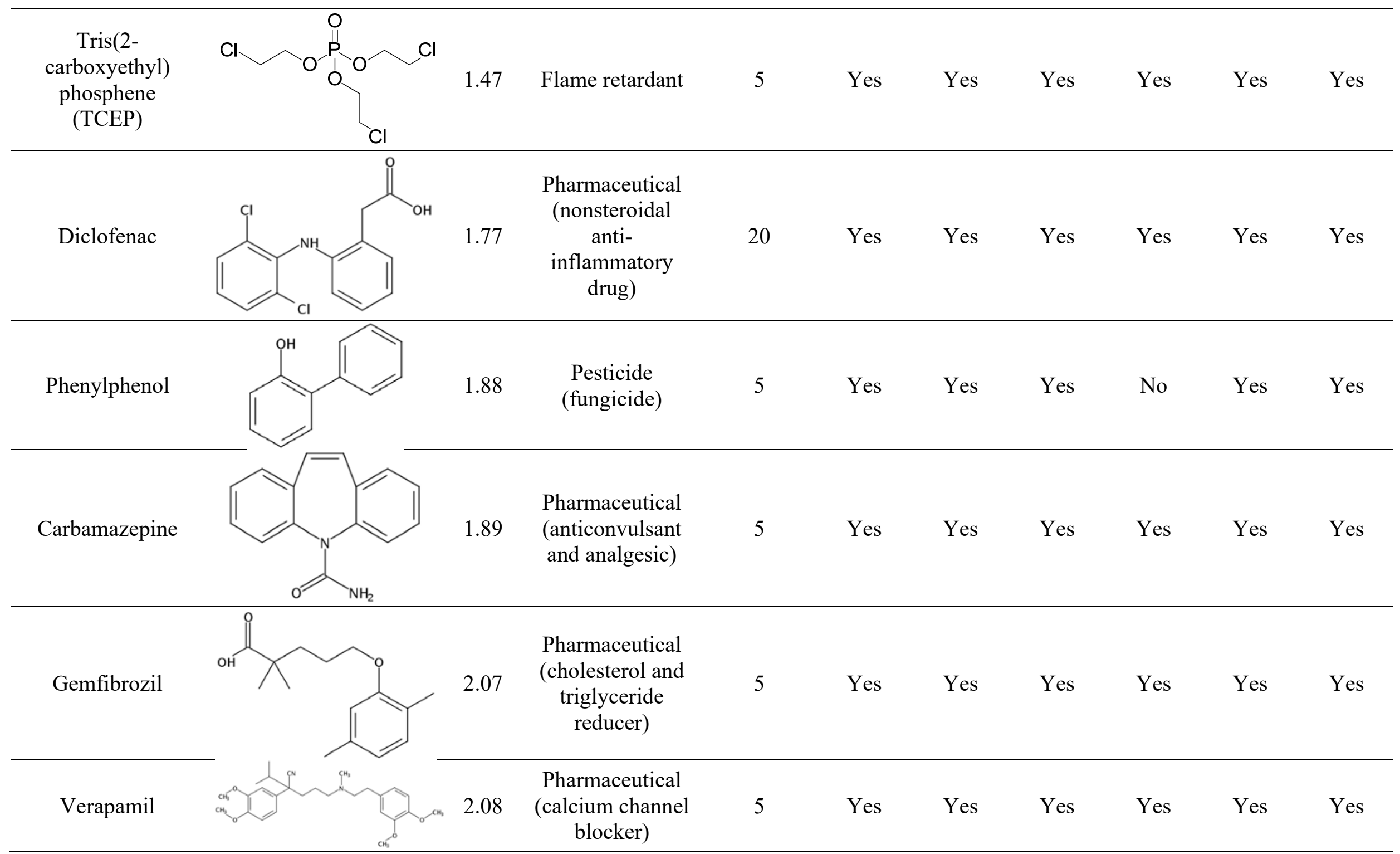




\begin{tabular}{|c|c|c|c|c|c|c|c|c|c|}
\hline Hydroxyzine & 2.15 & $\begin{array}{l}\text { Pharmaceutical } \\
\text { (antihistamine) }\end{array}$ & 5 & Yes & No & Yes & No & Yes & Yes \\
\hline Amitriptyline & 2.28 & $\begin{array}{l}\text { Pharmaceutical } \\
\text { (antidepressant) }\end{array}$ & 20 & Yes & Yes & Yes & Yes & Yes & Yes \\
\hline Simazine & 2.28 & $\begin{array}{l}\text { Pesticide } \\
\text { (herbicide) }\end{array}$ & 5 & Yes & Yes & Yes & No & Yes & No \\
\hline Omeprazole & 2.35 & $\begin{array}{l}\text { Pharmaceutical } \\
\text { (anti- } \\
\text { gastroesophaegal } \\
\text { reflux) }\end{array}$ & 20 & Yes & No & Yes & No & Yes & No \\
\hline Estriol & 2.53 & Hormone & 5 & Yes & Yes & Yes & Yes & Yes & Yes \\
\hline Atrazine & 2.64 & $\begin{array}{l}\text { Pesticide } \\
\text { (herbicide) }\end{array}$ & 5 & Yes & No & Yes & No & Yes & No \\
\hline
\end{tabular}




\begin{tabular}{|c|c|c|c|c|c|c|c|c|c|}
\hline Diuron & 2.68 & $\begin{array}{l}\text { Pesticide } \\
\text { (herbicide) }\end{array}$ & 5 & Yes & Yes & Yes & Yes & Yes & Yes \\
\hline Androstenedione & 2.72 & Hormone & 5 & Yes & Yes & Yes & Yes & Yes & Yes \\
\hline Diazepam & 2.80 & $\begin{array}{l}\text { Pharmaceutical } \\
\text { (muscle relaxant) }\end{array}$ & 5 & Yes & No & Yes & No & Yes & Yes \\
\hline Linuron & 3.12 & $\begin{array}{l}\text { Pesticide } \\
\text { (herbicide) }\end{array}$ & 5 & Yes & No & Yes & No & Yes & No \\
\hline
\end{tabular}




\begin{tabular}{|c|c|c|c|c|c|c|c|c|c|}
\hline Estrone & 3.13 & Hormone & 5 & Yes & Yes & Yes & Yes & Yes & Yes \\
\hline Clozapine & 3.23 & $\begin{array}{l}\text { Pharmaceutical } \\
\text { (antipsychosis) }\end{array}$ & 5 & Yes & Yes & Yes & Yes & Yes & Yes \\
\hline Butylparaben & 3.38 & $\begin{array}{l}\text { Personal care } \\
\text { product } \\
\text { formulation }\end{array}$ & 20 & No & - & Yes & Yes & Yes & Yes \\
\hline Bisphenol A & 3.64 & $\begin{array}{c}\text { Industrial } \\
\text { chemical (plastic } \\
\text { production) }\end{array}$ & 5 & Yes & Yes & Yes & Yes & Yes & Yes \\
\hline
\end{tabular}




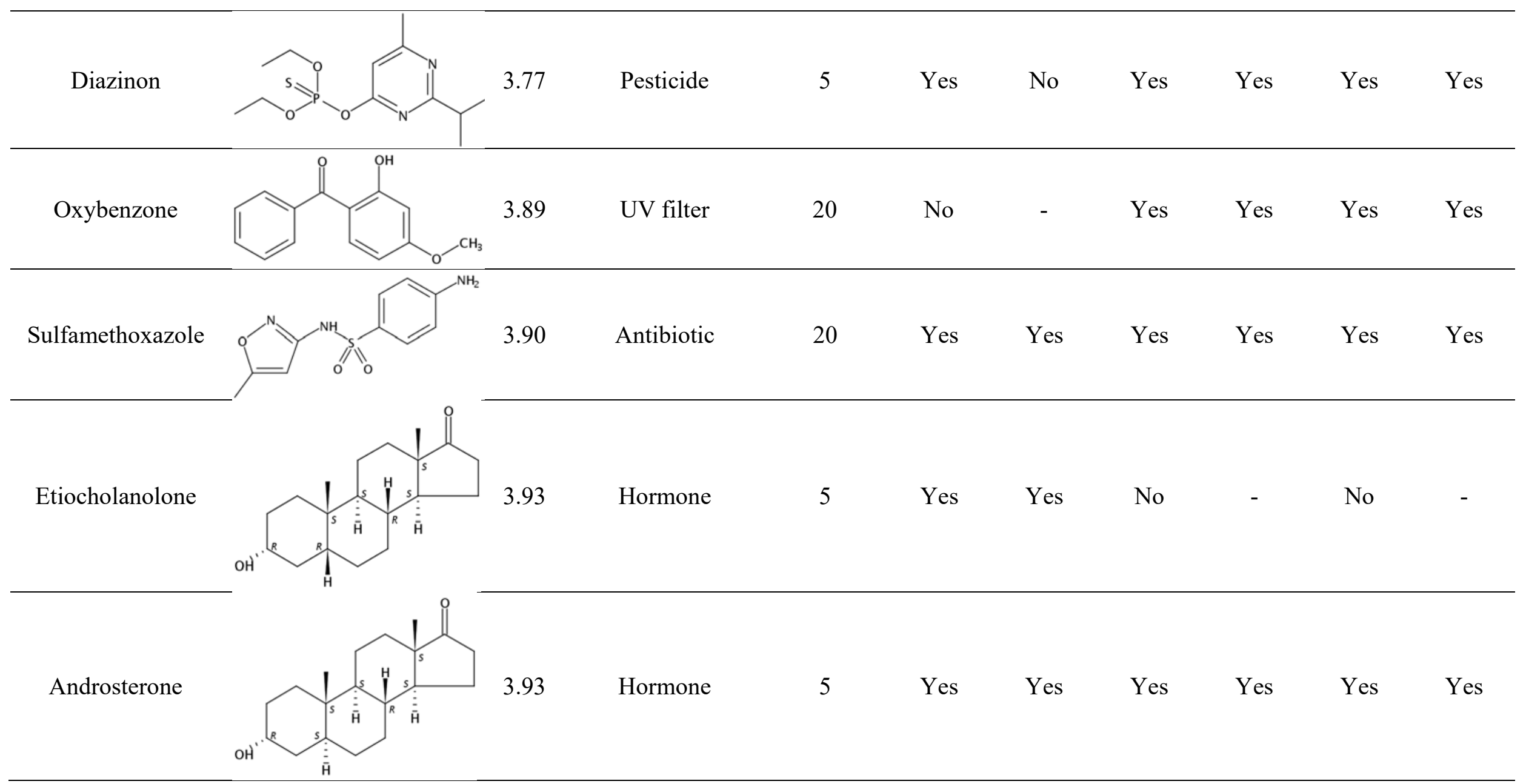




\begin{tabular}{|c|c|c|c|c|c|c|c|c|c|}
\hline $\begin{array}{c}\text { Di- } \\
\text { hydrotestosterone }\end{array}$ & 3.93 & Hormone & 5 & Yes & No & Yes & Yes & Yes & Yes \\
\hline Ethynylestradiol & 4.11 & Xenoestrogen & 5 & Yes & Yes & Yes & Yes & Yes & Yes \\
\hline $17 \alpha$-estradiol & 4.15 & Hormone & 5 & Yes & No & Yes & Yes & Yes & No \\
\hline $17 \beta$-estradiol & 4.15 & Hormone & 5 & Yes & Yes & Yes & Yes & Yes & Yes \\
\hline
\end{tabular}




\begin{tabular}{|c|c|c|c|c|c|c|c|c|c|}
\hline Chlorpyrifos & 5.00 & Pesticide & 5 & No & - & Yes & No & Yes & No \\
\hline Triclosan & 5.15 & Antibiotic & 20 & Yes & Yes & Yes & Yes & Yes & Yes \\
\hline 4-tert-Octylphenol & 5.18 & $\begin{array}{l}\text { Industrial } \\
\text { surfactant }\end{array}$ & 5 & No & - & Yes & Yes & Yes & Yes \\
\hline Fenofibrate & 5.80 & $\begin{array}{l}\text { Pharmaceutical } \\
\text { (cholesterol and } \\
\text { triglyceride } \\
\text { reducer) }\end{array}$ & 20 & No & - & Yes & Yes & Yes & Yes \\
\hline Triclocarban & & $\begin{array}{l}\text { Industrial } \\
\text { surfactant }\end{array}$ & 20 & Yes & Yes & Yes & Yes & Yes & Yes \\
\hline
\end{tabular}


Table S4. Estimation of TrOC concentration entering the external aerobic/anoxic and anoxic reactors and the control aerobic digester.
To analyse the biodegradation and sorption of TrOCs under aerobic/anoxic treatment, the TrOC concentration of sludge (in ng/L) going in to the reactor ( $Y_{\text {in-arobic/anoxic }}$ ) was estimated based on sludge flows from SBRosa (YSBRosA $)$ and anoxic reactor ( $\left.\mathrm{Y}_{\text {anoxic }}\right)$ :

$$
Y_{\text {in-aerobic/anoxic }}=Y_{S B R_{O S A}}+Y_{\text {anx }}
$$
$Y_{\text {in-aerobic/anoxic }}$

$$
=\frac{\left[\left(A_{S B R_{O S A}}+S_{S B R_{O S A}} \times M L S S_{S B R_{O S A}}\right) \times q_{1}+\left(A_{\text {anoxic }}+S_{\text {anoxic }} \times M L S S_{\text {anoxic }}\right) \times q_{4}\right]}{\left(q_{1}+q_{4}\right)}
$$

Where $\mathrm{A}$ and $\mathrm{S}$ were the aqueous and solid phase TrOC concentration of sludge, MLSS was the sludge concentration, $\mathrm{q}_{1}$ was the flow rate of sludge from SBRosA to aerobic/anoxic reactor (Figure 1a), and $\mathrm{q}_{4}$ was the flow rate of sludge from anoxic to aerobic/anoxic reactor (Figure 1a).

Likewise, the TrOC concentration of sludge going in to the anoxic reactor was estimated based on sludge flow from the aerobic/anoxic reactor ( $Y_{\text {aerobic/anoxic }}$ ):

$$
\begin{gathered}
Y_{\text {in-anoxic }}=Y_{\text {aerobic/anoxic }} \\
Y_{\text {in-anoxic }}=A_{\text {aerobic/anoxic }}+S_{\text {aerobic/anoxic }} \times M L S S_{\text {aerobic/anoxic }}
\end{gathered}
$$

Notably, the flow rate of sludge from the aerobic/anoxic to the anoxic reactor $\left(\mathrm{q}_{3}\right)$ was equal to the rate at which sludge was withdrawn from the anoxic reactor $\left(q_{4}+q_{5}\right)$ (Figure 1a).

The TrOC concentration of sludge going in to the aerobic digester was also estimated based on sludge flow from $\mathrm{SBR}_{\text {control: }}$

$$
\begin{gathered}
Y_{\text {in-aerobic }}=Y_{S B R_{\text {control }}} \\
Y_{\text {in-aerobic }}=A_{S B R_{\text {control }}}+S_{S B R_{\text {control }}} \times M L S S_{S B R_{\text {control }}}
\end{gathered}
$$




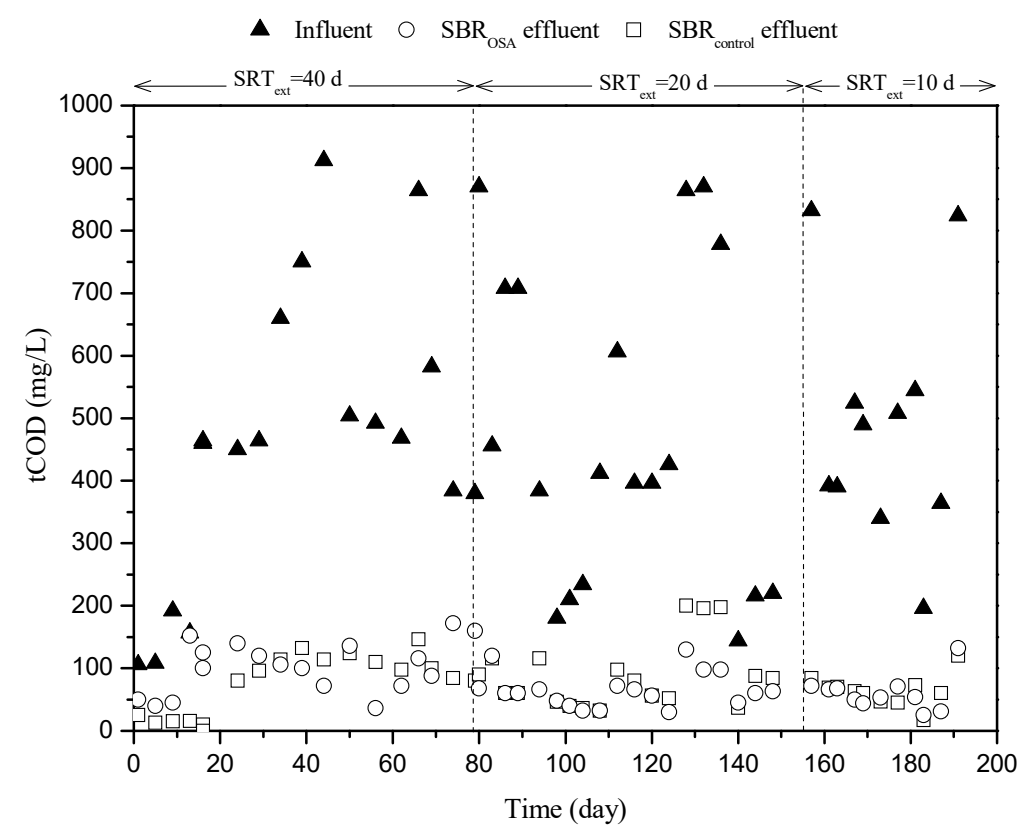

Figure S5. tCOD of SBRosa and SBR control when SRTsBR was 10 days and SRText was varied (10$40 \mathrm{~d})$. The dashed line indicates different $\mathrm{SRT}_{\text {ext. }}$

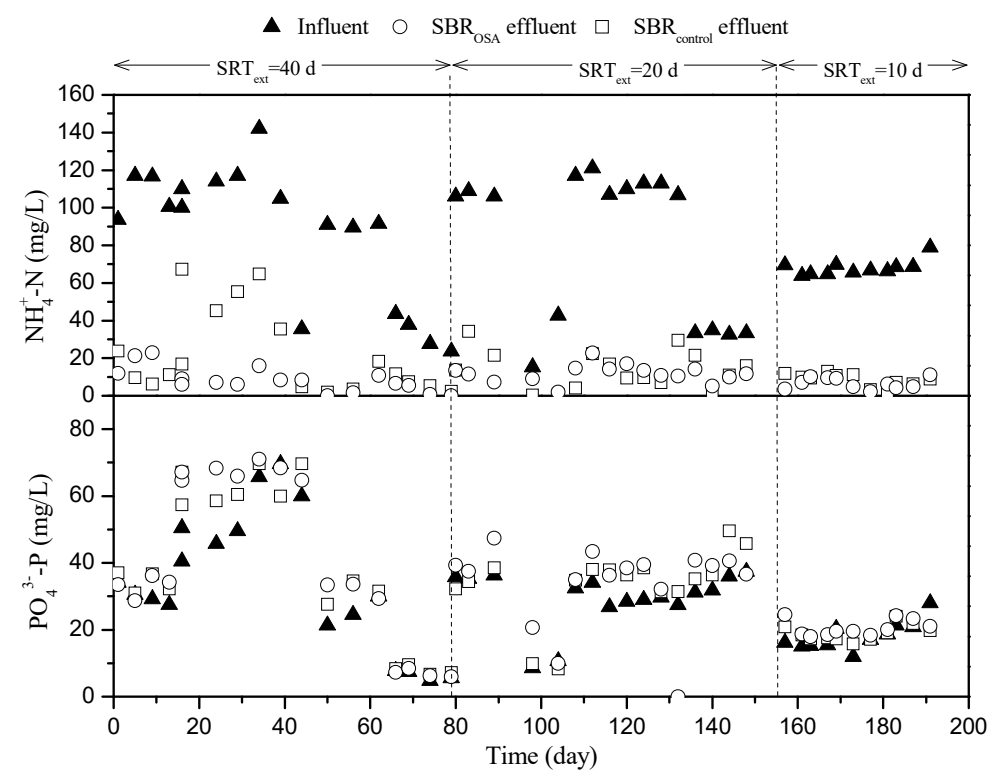

Figure S6. Ammonia and orthophosphate concentration of SBR control and SBRosa when SRTsBR was 10 days and $\mathrm{SRT}_{\text {ext.reactors }}$ was varied $(10-40 \mathrm{~d})$. The dashed line indicates different $\mathrm{SRT}_{\text {ext. }}$ 


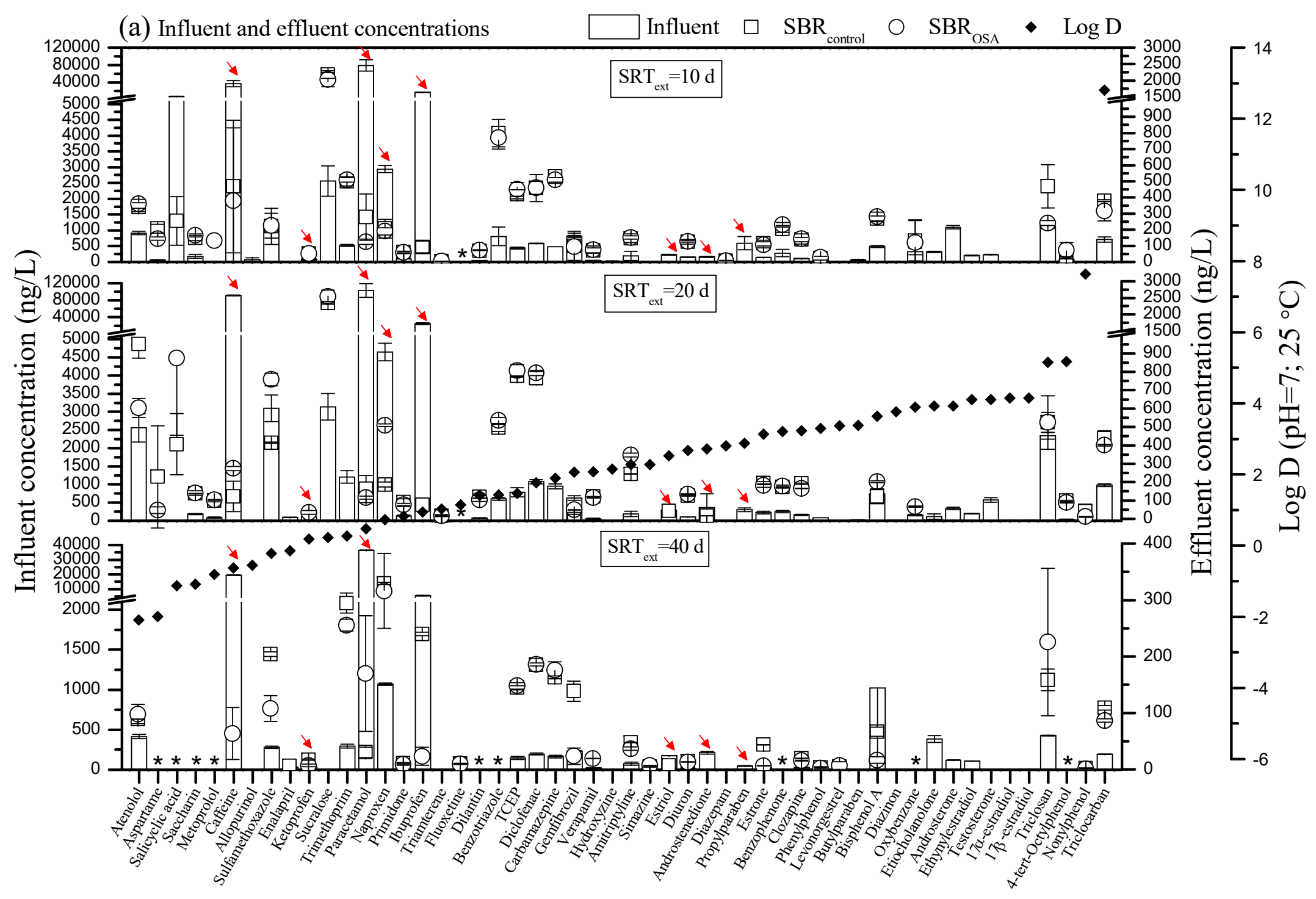




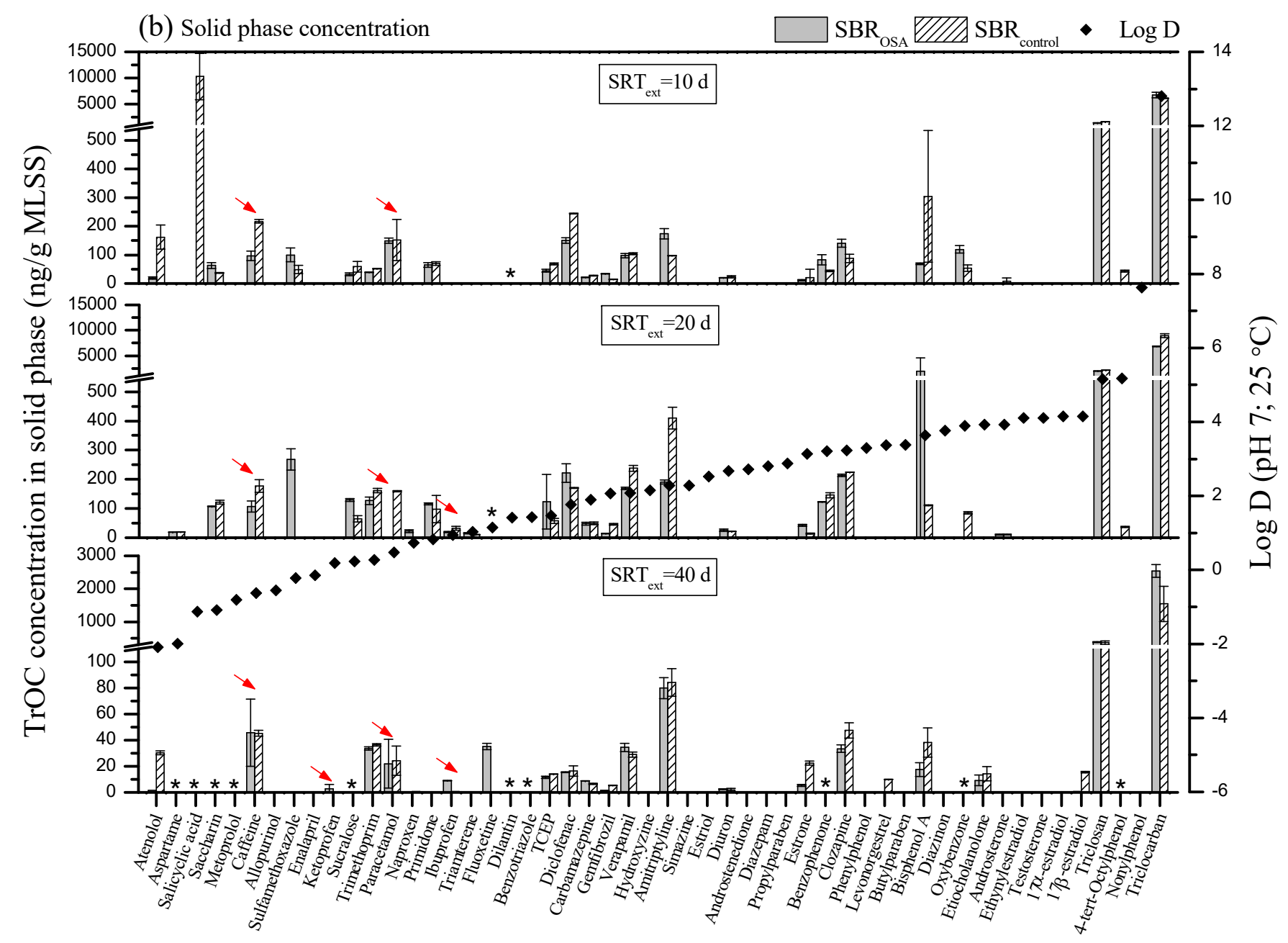

Figure S7. TrOC concentrations in the (a) influent, effluent, and (b) solid phase of sludge of SBRosa and SBR control when SRTsBR was maintained at $10 \mathrm{~d}$ and SRT ext was varied $(10-40 \mathrm{~d})$. The values are the average of two measurements $(n=2)$. The asterisks $(*)$ represent TrOCs that were not analysed in a particular sampling campaign. The arrows $(\rightarrow)$ denote contaminants that were highly biodegraded. 
Table S8. TrOCs with notable variation ( $>30 \%$ difference) in SBRosa and SBR control effluents when SRTSBR was maintained at $10 \mathrm{~d}$ and SRText was varied (10-40 d); The values are the average of two measurements $(n=2)$.

\begin{tabular}{|c|c|c|c|c|}
\hline \multirow{2}{*}{$\mathrm{SRT}_{\text {ext }}$ (d) } & \multirow{2}{*}{ TrOC } & \multirow{2}{*}{ Influent (ng/L) } & \multicolumn{2}{|c|}{ Effluent (ng/L) } \\
\hline & & & SBR OSA & SBR $_{\text {control }}$ \\
\hline \multirow{4}{*}{10} & Aspartame & $63 \pm 6$ & $145 \pm 11$ & $207 \pm 27$ \\
\hline & Paracetamol & $79,000 \pm 12,728$ & $125 \pm 16$ & $278 \pm 144$ \\
\hline & Triclosan & $1,126 \pm 71$ & $241 \pm 16$ & $469 \pm 134$ \\
\hline & 4-tert-octylphenol & $73 \pm 35$ & $73 \pm 48$ & $26 \pm 7$ \\
\hline \multirow{14}{*}{20} & Atenolol & $2,560 \pm 396$ & $604 \pm 51$ & $952 \pm 76$ \\
\hline & Aspartame & $20 \pm 0$ & $48 \pm 19$ & $229 \pm 287$ \\
\hline & Salicylic acid & $\begin{array}{c}\text { Below detection } \\
\text { limit }\end{array}$ & $874 \pm 419$ & $406 \pm 167$ \\
\hline & Caffeine & $91,500 \pm 707$ & $274 \pm 11$ & $122 \pm 82$ \\
\hline & Ketoprofen & $107 \pm 4$ & $35 \pm 11$ & $\begin{array}{l}\text { Below detection } \\
\text { limit }\end{array}$ \\
\hline & Paracetamol & $103,100 \pm 16,263$ & $116 \pm 8$ & $163 \pm 73$ \\
\hline & Naproxen & $4,650 \pm 240$ & $509 \pm 7$ & $187 \pm 13$ \\
\hline & Ibuprofen & $24,700 \pm 2,121$ & $\begin{array}{c}\text { Below detection } \\
\text { limit }\end{array}$ & $77 \pm 0$ \\
\hline & Estriol & $291 \pm 25$ & $\begin{array}{c}\text { Below detection } \\
\text { limit }\end{array}$ & $43 \pm 0$ \\
\hline & Androstenedione & $368 \pm 374$ & $\begin{array}{c}\text { Below detection } \\
\text { limit }\end{array}$ & $17 \pm 0$ \\
\hline & Bisphenol A & $745 \pm 1$ & $202 \pm 6$ & $123 \pm 40$ \\
\hline & Oxybenzone & $161 \pm 20$ & $66 \pm 3$ & $\begin{array}{l}\text { Below detection } \\
\text { limit }\end{array}$ \\
\hline & Sulfamethoxazole & $3,100 \pm 368$ & $758 \pm 34$ & $414 \pm 3$ \\
\hline & Nonylphenol & $20 \pm 1$ & $13 \pm 0$ & $44 \pm 36$ \\
\hline \multirow{10}{*}{40} & Caffeine & $19,500 \pm 283$ & $63 \pm 46$ & $563 \pm 26$ \\
\hline & Ketoprofen & $32 \pm 4$ & $7 \pm 2$ & $17 \pm 1$ \\
\hline & Paracetamol & $36,450 \pm 70$ & $170 \pm 102$ & $30 \pm 9$ \\
\hline & Ibuprofen & $5,525 \pm 247$ & $23 \pm 16$ & $240 \pm 3$ \\
\hline & Gemfibrozil & $232 \pm 4$ & $23 \pm 15$ & $138 \pm 17$ \\
\hline & Estriol & $175 \pm 0$ & $\begin{array}{l}\text { Below detection } \\
\text { limit }\end{array}$ & $7 \pm 0$ \\
\hline & Estrone & $32 \pm 0$ & $6 \pm 1$ & $43 \pm 1$ \\
\hline & Bisphenol A & $1,020 \pm 1203$ & $16 \pm 6$ & $67 \pm 8$ \\
\hline & Sulfamethoxazole & $280 \pm 13$ & $107 \pm 23$ & $204 \pm 4$ \\
\hline & Triclosan & $428 \pm 5$ & $226 \pm 131$ & $158 \pm 19$ \\
\hline
\end{tabular}


Table S9. Flow rate and change in receiving media during sludge interchange in the OSA system at different external reactor $\mathrm{SRT}_{\mathrm{ext}}$

\begin{tabular}{|c|c|c|c|c|c|}
\hline $\mathrm{SRT}_{\text {ext }}(\mathrm{d})$ & $\begin{array}{l}\mathrm{q}_{1}(\mathrm{~mL} / \mathrm{d})^{\mathrm{a}} / \\
\Delta \text { receiving } \\
\text { media }(\%)^{b}\end{array}$ & $\begin{array}{l}\mathrm{q}_{2}(\mathrm{~mL} / \mathrm{d})^{\mathrm{c}} / \\
\Delta \text { receiving } \\
\text { media }(\%)^{b}\end{array}$ & $q_{3}{ }^{d}(m L / d)$ & $\begin{array}{l}\mathrm{q}_{4}^{\mathrm{e}}(\mathrm{mL} / \mathrm{d}) / \\
\Delta \text { receiving } \\
\text { media }(\%)^{b}\end{array}$ & $\begin{array}{l}q_{5}(\mathrm{~mL} / \mathrm{d})^{\mathrm{f}} / \\
\Delta \text { receiving } \\
\text { media }(\%)^{b}\end{array}$ \\
\hline 10 & $468 / 23.4$ & $200 / 10$ & 400 & $132 / 6.6$ & $68 / 1.4 \%$ \\
\hline 20 & $234 / 11.7$ & $100 / 5$ & 200 & $66 / 3.3$ & $34 / 0.7 \%$ \\
\hline 40 & $117 / 5.9 \%$ & $50 / 2.5$ & 100 & $33 / 1.7$ & $17 / 0.4 \%$ \\
\hline
\end{tabular}

${ }^{\mathrm{a}} \mathrm{q}_{1}=$ SBRosA to aerobic/anoxic

${ }^{\mathrm{b}} \Delta$ receiving media $(\%)=$ volume transferred to the reactor/total volume of the reactor $\mathrm{x} 100$

${ }^{\mathrm{c}} \mathrm{q}_{2}=$ aerobic/anoxic to anoxic

${ }^{\mathrm{d}} \mathrm{q}_{3}=$ wasted from aerobic/anoxic

${ }^{\mathrm{e}} \mathrm{q}_{4}=$ anoxic to aerobic/anoxic

${ }^{\mathrm{f}} \mathrm{q}_{5}=$ anoxic to SBROSA 
Table S10. TrOCs with notable variation ( $>30$ difference) in the solid phase of SBRosa and

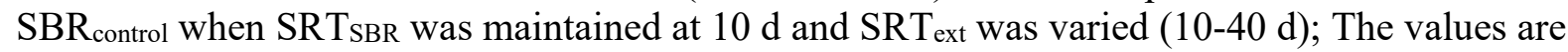
the average of two measurements $(n=2)$.

\begin{tabular}{|c|c|c|c|}
\hline $\mathrm{SRT}_{\text {ext }}(\mathrm{d})$ & TrOC & $\begin{array}{c}\text { SBRosA (ng/g dry } \\
\text { solids) }\end{array}$ & $\begin{array}{c}\text { SBR }_{\text {control }} \text { (ng/g dry } \\
\text { solids) }\end{array}$ \\
\hline \multirow{12}{*}{10} & TCEP & $45 \pm 5$ & $68 \pm 4$ \\
\hline & Atenolol & $19 \pm 42$ & $162 \pm 42$ \\
\hline & Salicylic acid & Below detection limit & $10,283 \pm 4,434$ \\
\hline & Caffeine & $97 \pm 16$ & $217 \pm 7$ \\
\hline & Diclofenac & $150 \pm 9$ & $244 \pm 2$ \\
\hline & Amtriptylene & $173 \pm 18$ & $97 \pm 1$ \\
\hline & Estrone & $11 \pm 2$ & $21 \pm 29$ \\
\hline & Benzophenone & $83 \pm 18$ & $44 \pm 3$ \\
\hline & Clozapine & $141 \pm 14$ & $88 \pm 15$ \\
\hline & Bisphenol A & $69 \pm 3$ & $304 \pm 230$ \\
\hline & Oxybenzone & $119 \pm 13$ & $53 \pm 11$ \\
\hline & Sulfamethoxazole & $100 \pm 24$ & $49 \pm 14$ \\
\hline \multirow{14}{*}{20} & TCEP & $123 \pm 93$ & $58 \pm 9$ \\
\hline & Caffeine & $107 \pm 19$ & $178 \pm 22$ \\
\hline & Sucralose & $129 \pm 5$ & $65 \pm 11$ \\
\hline & Paracetamol & Below detection limit & $159 \pm 2$ \\
\hline & Ibuprofen & $19 \pm 2$ & $33 \pm 17$ \\
\hline & Diclofenac & $222 \pm 32$ & $171 \pm 1$ \\
\hline & Gemfibrozil & $15 \pm 0$ & $46 \pm 3$ \\
\hline & Verapamil & $169 \pm 4$ & $238 \pm 10$ \\
\hline & Amtriptylene & $191 \pm 7$ & $410 \pm 37$ \\
\hline & Estrone & $43 \pm 3$ & $14 \pm 2$ \\
\hline & Bisphenol A & $1,922 \pm 2,620$ & $111 \pm 1$ \\
\hline & Oxybenzone & Below detection limit & $85 \pm 4$ \\
\hline & Sulfamethoxazole & $268 \pm 37$ & Below detection limit \\
\hline & Triclocarban & $6,810 \pm 91$ & $8,931 \pm 412$ \\
\hline \multirow{5}{*}{40} & Atenolol & Below detection limit & $30 \pm 1$ \\
\hline & Estrone & $5 \pm 1$ & $22 \pm 1$ \\
\hline & Clozapine & $33 \pm 3$ & $47 \pm 33$ \\
\hline & Bisphenol A & $17 \pm 5$ & $38 \pm 11$ \\
\hline & Triclocarban & $2543 \pm 195$ & $1,541 \pm 533$ \\
\hline
\end{tabular}




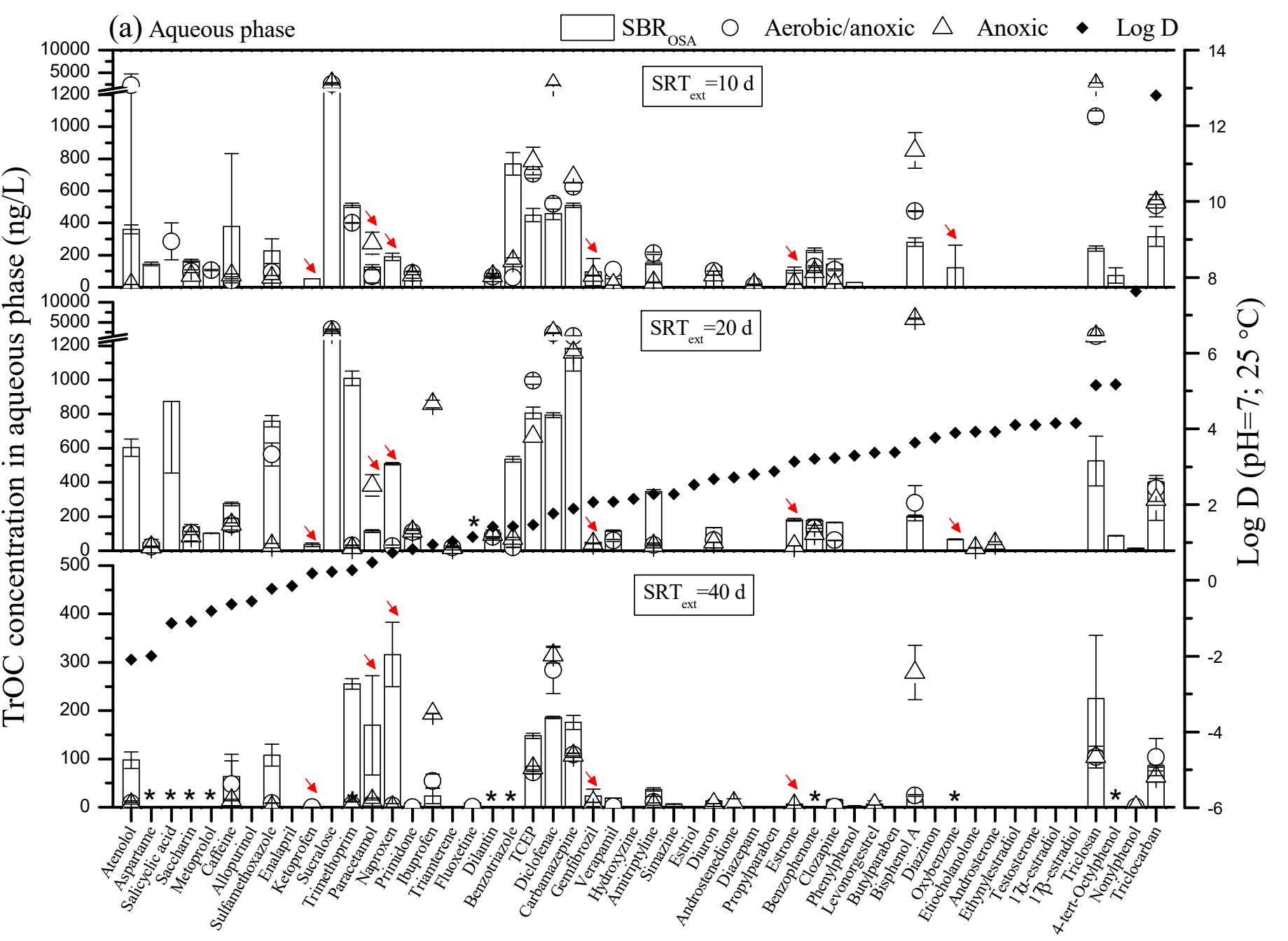




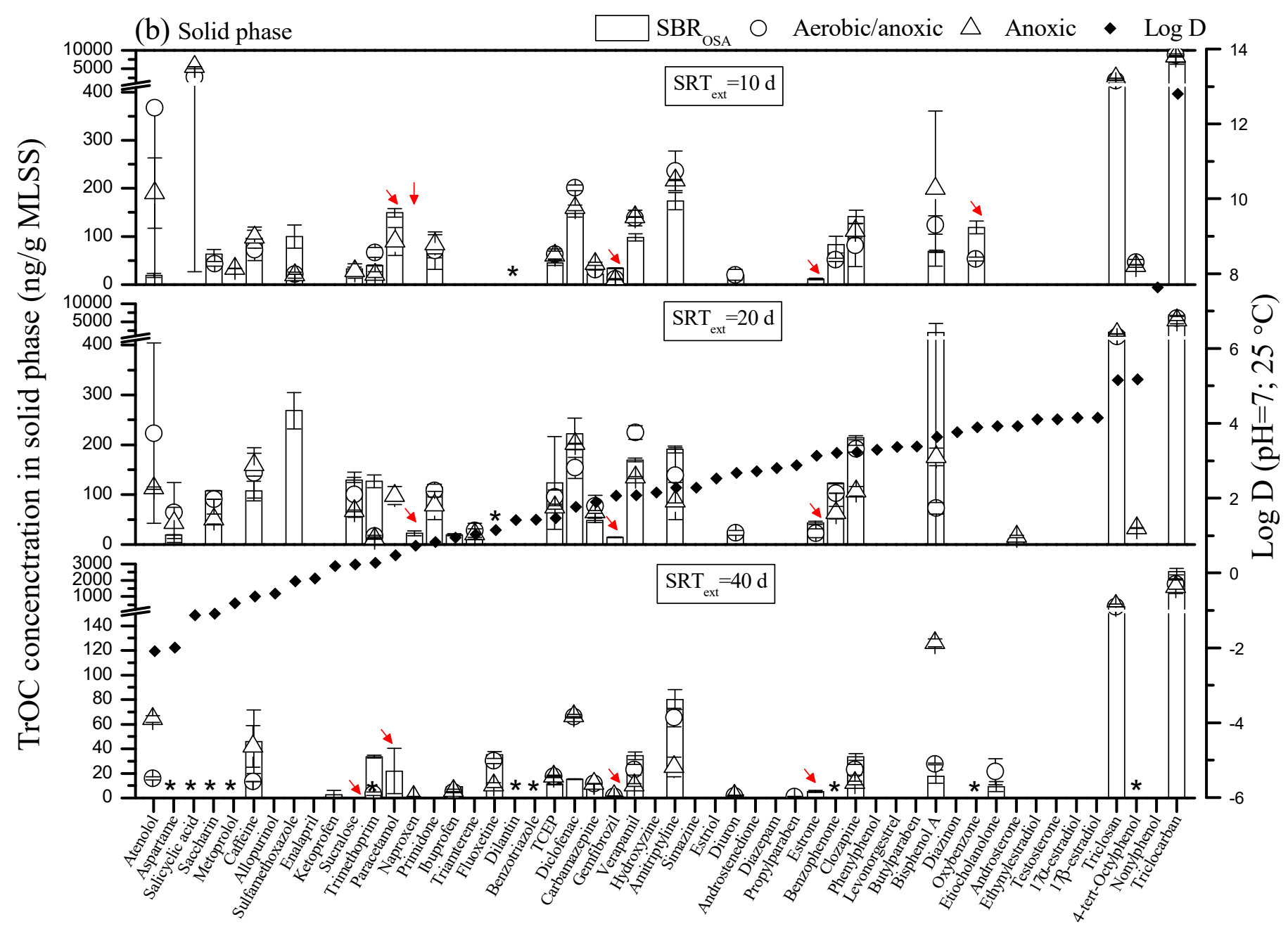

Figure S11. TrOC concentration in the (a) aqueous and (b) solid phase of sludge in the external aerobic/anoxic and anoxic reactor of OSA when SRTSBR was maintained at $10 \mathrm{~d}$ and SRText was varied (10-40 d). The values are the average of two measurements $(n=2)$.

The asterisks $(*)$ represent contaminants that were not analysed in a particular sampling campaign. The arrows $(\rightarrow)$ denote denote contaminants that were highly biodegraded in the aerobic/anoxic reactor. No contaminant was highly biodegraded in the anoxic reactor. 


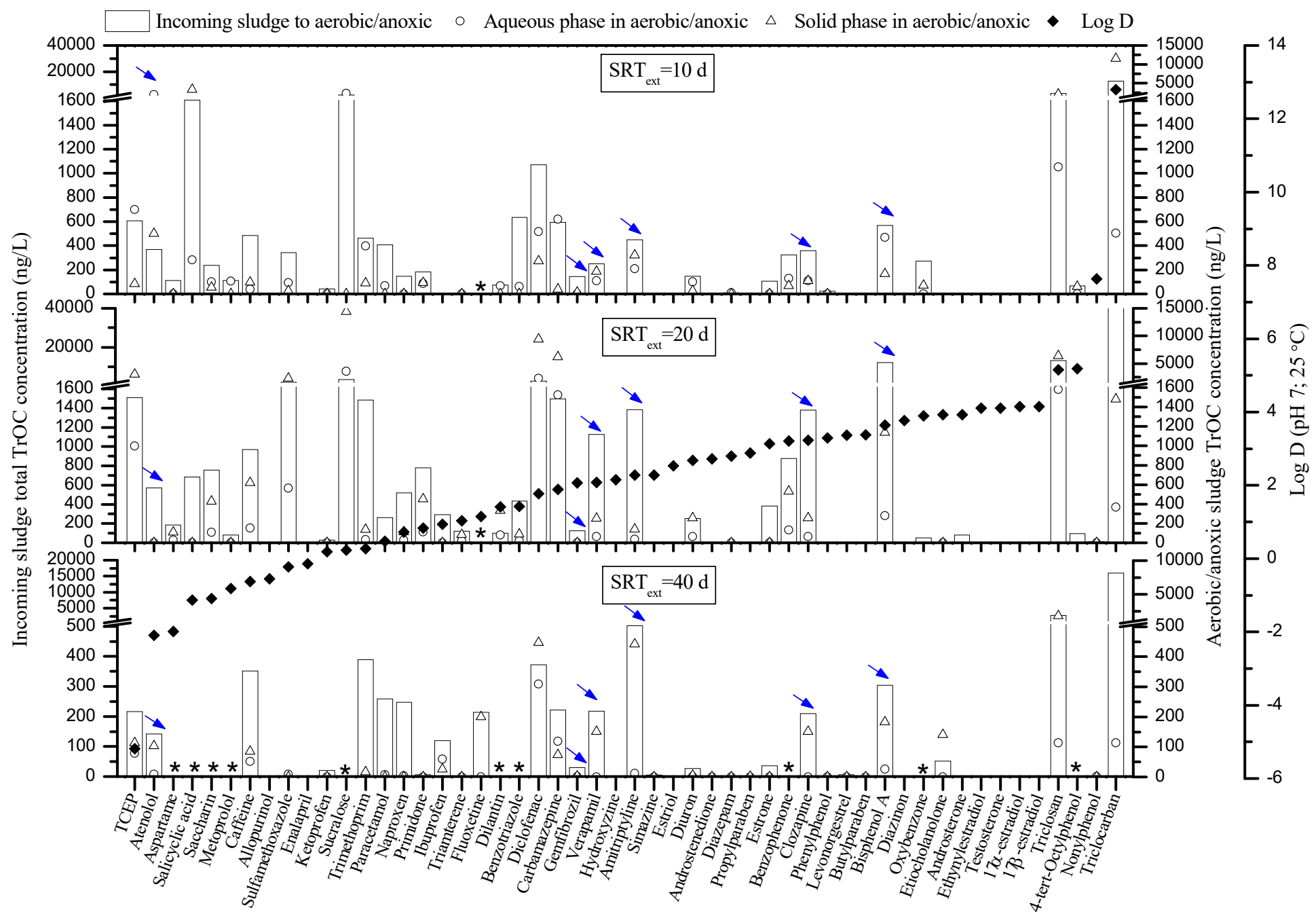

Table S12. The concentration TrOCs entering the aerobic/anoxic reactor (Yin-aerobic/anoxic, labelled as "incoming sludge") vs. the concentration of TrOCs in aqueous and solid phase of sludge in aerobic/anoxic reactor when SRTSBR was maintained at $10 \mathrm{~d}$ and SRT ext was varied (10-40 d). The values are the average of two measurements. The asterisks $(*)$ represent contaminants that were not analysed in a particular sampling campaign. The biodegradation of some TrOCs (denoted by arrows $\rightarrow$ ) increased when $\mathrm{SRT}_{\text {ext }}$ was increased from 10 to $20 \mathrm{~d}$, but decreased when SRText was further increased to $40 \mathrm{~d}$. 


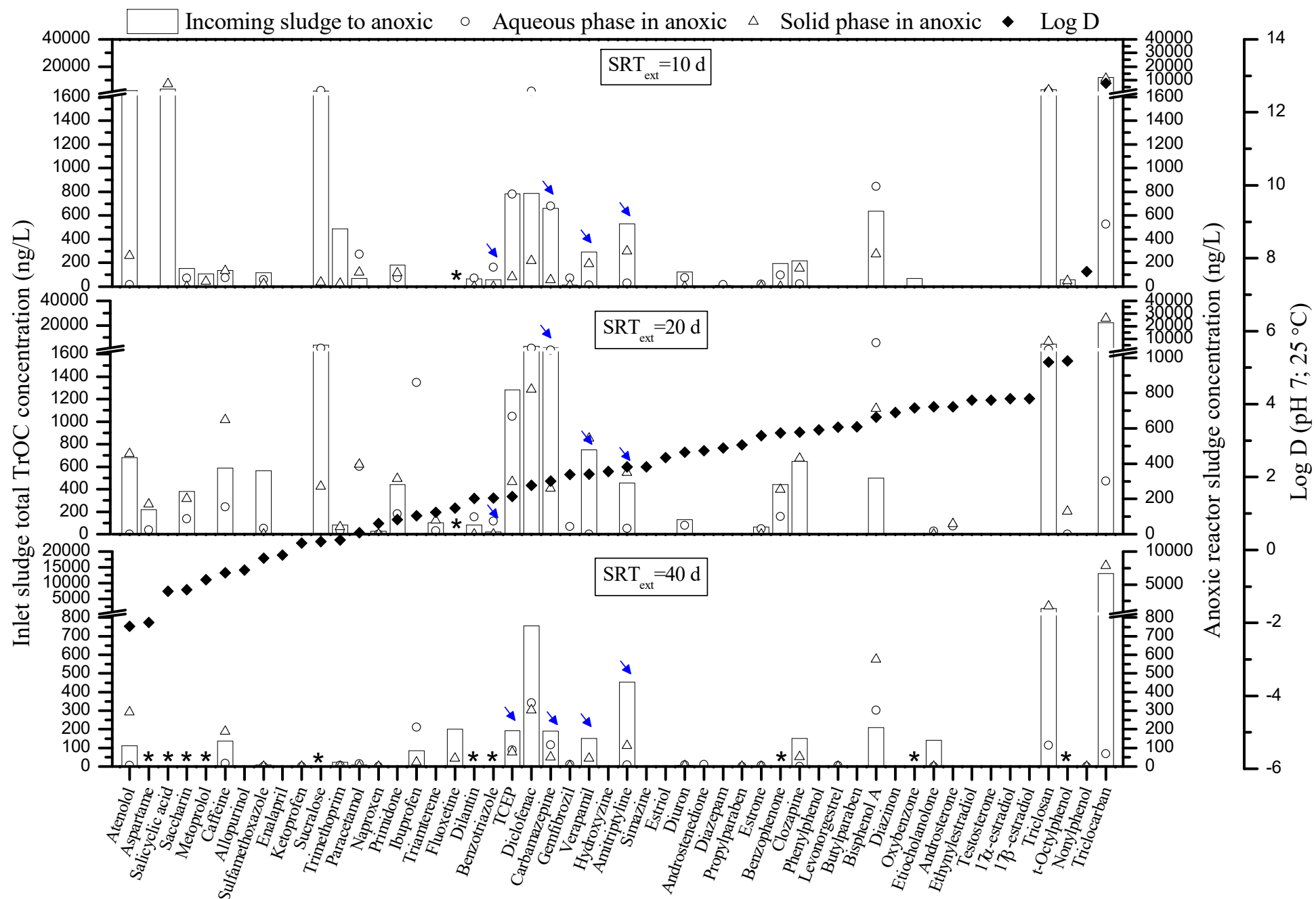

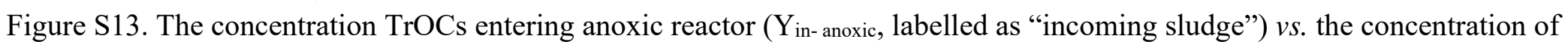
TrOCs in aqueous and solid phase of sludge in anoxic reactor when SRTSBR was maintained at $10 \mathrm{~d}$ and SRT ext was varied (10-40 d). The values are the average of two measurements. The asterisks $\left(^{*}\right)$ represent contaminants that were not analysed in a particular sampling campaign. The biodegradation of some contaminants (denoted by arrows $\rightarrow$ ) increased when $\mathrm{SRT}_{\text {ext }}$ was increased from 10 to $40 \mathrm{~d}$. 
(a) Aqueous phase

SBR

$\nabla$ Aerobic digester

- $\log \mathrm{D}$

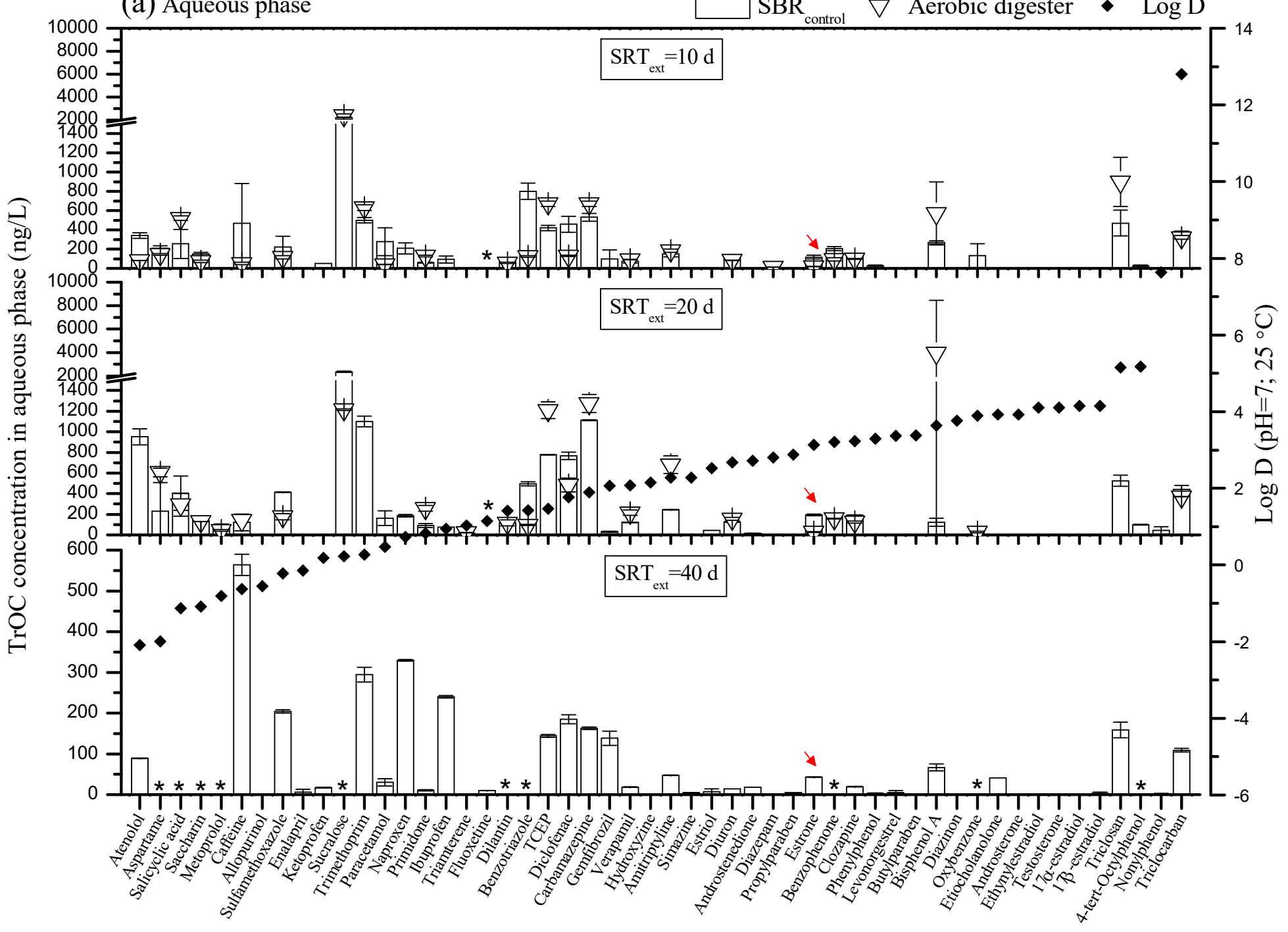


(a) Aqueous phase

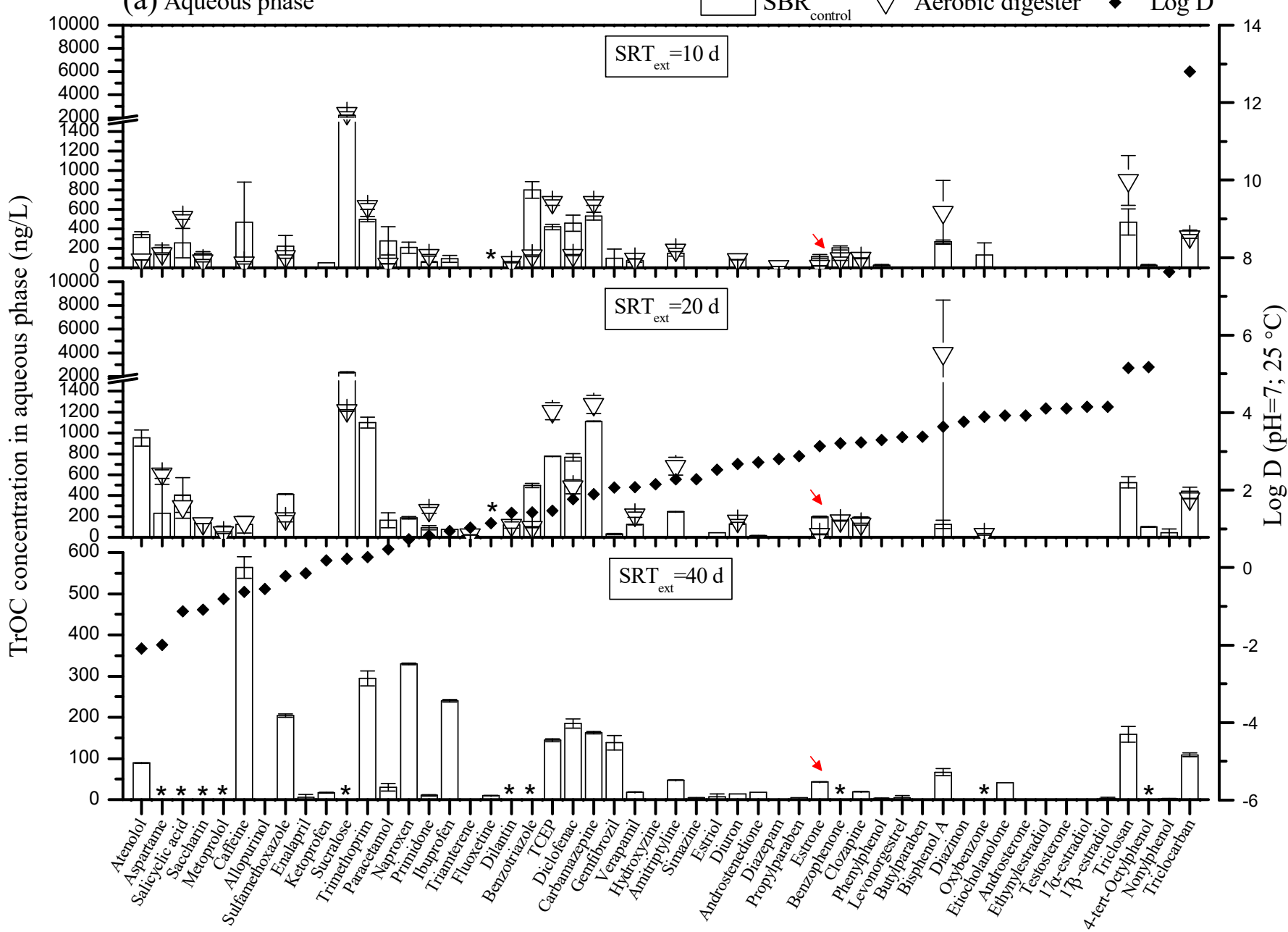

Figure S14. TrOC concentration in the (a) aqueous and (b) solid phase of sludge in the aerobic digester when SRTSBR was maintained at $10 \mathrm{~d}$ and SRText was varied $(10-40 \mathrm{~d})$. The values are the average of two measurements $(n=2)$. The asterisks $(*)$ represent contaminants that were not analysed in a particular sampling campaign. The arrows $(\rightarrow)$ denote contaminants that were highly biodegraded. 


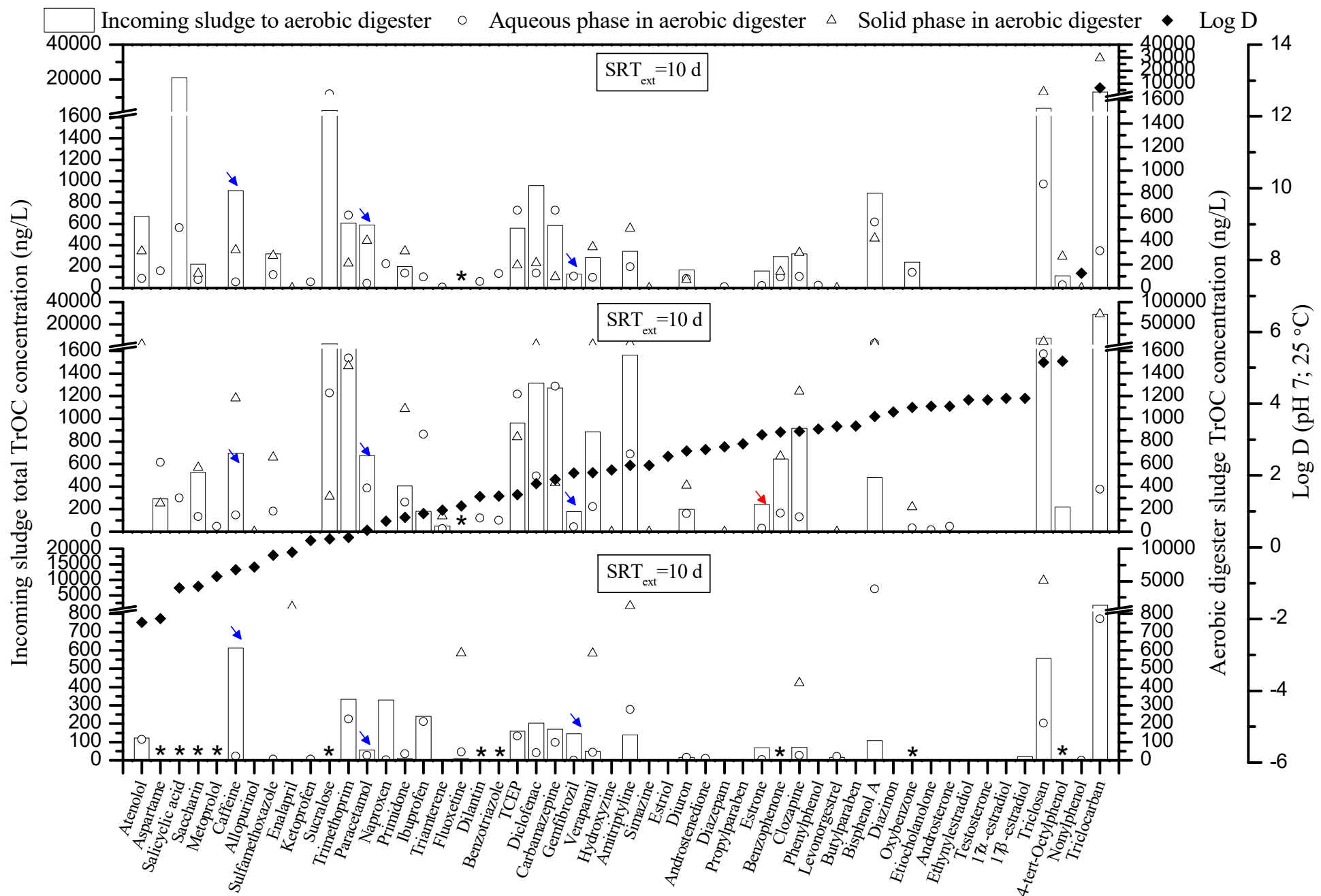

Figure S15. The concentration TrOCs entering control aerobic digester (Yin- aerobic, labelled as "incoming sludge") vs. the concentration of TrOCs in aqueous and solid phase of sludge in the aerobic digester when SRTSBR was maintained at $10 \mathrm{~d}$ and SRT ext was varied $(10-40 \mathrm{~d})$. The values are the average of two measurements. The asterisks $(*)$ represent contaminants that were not analysed in a particular sampling campaign. The biodegradation of some TrOCs (denoted by arrows $\rightarrow$ ) increased when SRText was increased from 10 to 40 . 